\title{
INTERNAÇÕES POR ABORTO ESPONTÂNEO: UM RETRATO DE SUA OCORRÉNCIA EM FORTALEZA
}

Camila Lima Ribeiro'1, Francisca de Oliveira Albuquerque², Adriano Rodrigues de Souza ${ }^{3}$

Objetivo: o estudo teve como objetivo caracterizar as internações por aborto espontâneo no município de Fortaleza, Ceará, no periodo de 2010 a 2014. Metodologia: estudo descritivo exploratório, com dados obtidos por meio do Departamento de Informática do Sistema Único de Saúde. Resultados: os dados mostram que foram registradas 14.203 internações por abortamentos espontâneos, sendo 2010 o ano de maior incidência. Observou-se ainda que a maioria dos atendimentos concentrou-se no sistema público de saúde e a maior parte deles em caráter de urgência. Conclusões: os dados permitem afirmar a urgência do acesso da população à educação sexual nas escolas.

Descritores: Aborto espontâneo, Saúde Reprodutiva, Epidemiologia.

\section{HOSPITALIZATION FROM MISCARRIAGES:A PORTRAIT OF ITS OCCURRENCE IN FORTALEZA.}

Objective: The study aimed to characterize hospitalizations from miscarriage in Fortaleza, Ceará, in the period 2010-2014. Methodology : A descriptive exploratory study, with data obtained from the Department of Health System Information. results: the data shows that 14,203 admissions were recorded from miscarriages, and 2010 being the year of highest incidence. It was also observed that most of the visits focused in the public health system and most of them on an urgent basis. Conclusions: The data allows us to state the urgency of sex education in schools for the population.

Descriptors: Abortion Spontaneous, Reproductive health, Epidemiology.

\section{HOSPITALIZACIÓN POR ABORTO ESPONTÁNEO : UN RETRATO DE SU APARICIÓN EN FORTALEZA.}

Objetivo: El objetivo del estudio fue caracterizar las hospitalizaciones por aborto involuntario en Fortaleza, Ceará, en el período 2010-2014.Metodología: Un estudio exploratorio descriptivo, con datos obtenidos del Departamento de Información del Sistema de Salud . resultados : los datos muestran que 14,203 admisiones fueron registrados por abortos espontáneos , y 2010 - el año de mayor incidencia. También se observó que la mayoría de las visitas se centraron en el sistema de salud pública y la mayoría de ellos con carácter de urgencia. Conclusiones : Los datos permiten afirmar la urgencia de acceso de la población a la educación sexual en las escuelas.

Descriptores: Aborto Espontáneo, Salud Reproductiva, Epidemiología. 


\section{INTRODUÇÃO}

Etimologicamente, aborto, do latim ab (privação) e ortus (nascimento), significa o impedimento do nascimento. A Organização Mundial de Saúde (OMS) e a Federação Internacional de Ginecologia e Obstetrícia (FIGO) definem o aborto como a interrupção espontânea ou induzida da gravidez antes da viabilidade do produto da concepção, correspondendo à perda do concepto até a $20^{\underline{a}}$ e a $22^{\underline{a}}$ semanas completas e/ou com feto de até $500 \mathrm{~g}^{(1)}$. É considerado espontâneo, quando se inicia independentemente de qualquer procedimento ou mecanismo externo. É considerado provocado, quando resulta da utilização de qualquer processo abortivo externo, químico ou mecânico. Esse pode ter motivação voluntária ou involuntária da gestante, e ser considerado legal ou ilegal(2).

Conforme a legislação vigente no Brasil - Decretolei 2848 (Brasil, 1940) -, o aborto é considerado crime. Entretanto, de acordo com o artigo 128 do Código Penal, a gestante que sofrer risco de morte e engravidar a partir de uma violação sexual está autorizada a interromper a gravidez (Brasil, 1940). Além disso, em 2012, foi julgada pelo Superior Tribunal Federal (STF) a descriminalização do aborto em casos de fetos anencefálicos, alterando o artigo 128 do Código Penal ${ }^{(3)}$.

A proporção mundial de abortamentos é de cerca de 50 milhões de casos por ano.

No Brasil, o número de aborto é cerca de 1,4 milhão por ano, o que refere uma relação de 23 abortos a cada 100 gestações. A incidência de óbitos por complicações de abortos oscila em torno de $12 \%$ do total de óbitos, ocupando o terceiro lugar entre suas causas ${ }^{(4)}$.

Além das complicações psicológicas resultantes do aborto, as complicações físicas mais frequentes são: hemorragia, retenção de resto de placenta, infecção, perfuração do útero e, com menor incidência, peritonite, tétano, septicemia, inclusive esterilidade ${ }^{(5)}$.

Levando-se em consideração que a curetagem uterina é um dos procedimentos obstétricos mais realizados no país, sendo uma das causas no aumento dos índices de mortalidade feminina, e responsável por várias complicações físicas e psicológicas, além da inexistência de dados e registros desse agravo no estado do Ceará e no município de Fortaleza, decidiu-se realizar este estudo, perguntamo-nos: qual a faixa etária das mulheres que apresentaram aborto espontâneo? Qual a unidade hospitalar de maior ocorrência? Que gastos registram?

Objetivou-se caracterizar as internações por aborto espontâneo no municipio de Fortaleza.

O estudo proporcionará aos gestores e administradores públicos e hospitalares conhecer a situação e o perfil das internações por abortos espontâneos no município de Fortaleza.

Como contribuição do estudo, podemos ressaltar que o conhecimento do perfil epidemiológico das internações por abortos espontâneos contribuirá para tomada de decisão por parte da gestão pública, junto à rede de assistência à saúde da mulher, contribuindo para a estruturação de consultas de pré-natal, pós-natal e de avaliação materna pela implementação de medidas que venham a reduzir e/ou controlar essa patologia.

\section{METODOLOGIA}

Trata-se de um estudo
descritivo exploratório,
de caráter quantitativo.
Os dados da pesquisa foram coletados no site do Departamento de Informática do SUS (DATASUS), nos meses de abril e maio de 2015 . Primeiramente, selecionamos as variáveis: município e aborto espontâneo, identificando que os dados apresentavam números significativos. A população da pesquisa foi composta por 14.203

ocorrências de aborto espontâneo, que foram atendidas ou tiveram registro na rede de assistência de saúde de Fortaleza, entre os anos de 2010 a 2014. A coleta ocorreu com o tabulador de internet (TABNET), existente na página do DATASUS.

Realizamos as tabulações dos seguintes dados: internações por faixa etária, segundo ano, caráter e regime do atendimento, dias de permanência, custos do internamento hospitalar e óbitos ocorridos.

Os custos das Autorizações por Internamento Hospitalar (AIH) foram coletados a partir do Sistema de Gerenciamento da Tabela de Procedimentos, Medicamentos, Órteses, Próteses e Materiais Especiais (SIGTAP) do DATASUS.

Os dados coletados foram digitados e tabulados por meio do programa Excel, possibilitando realizar a análise descritiva quanto ao tempo, lugar e pessoa dessa patologia. 
A pesquisa focou dados secundários e de domínio público, fato que possibilita a não apresentação do projeto de pesquisa junto aos comitês de ética.

\section{RESULTADOS}

Os dados coletados foram representados e estruturados através de tabela e gráfico a seguir.

Tabela 1 - Distribuição das variáveis de abortos espontâneos ocorridos no município de Fortaleza. Fortaleza, 2010 a 2014.

\begin{tabular}{|c|c|c|c|c|c|c|c|c|c|c|c|c|}
\hline \multirow{2}{*}{$\begin{array}{l}\text { Variáueis } \\
\text { FAIXA ETÁRIA }\end{array}$} & \multicolumn{12}{|c|}{ ANOS } \\
\hline & 2010 & $\%$ & 2011 & $\%$ & 2012 & $\%$ & 2013 & $\%$ & 2014 & $\%$ & Total & $\%$ \\
\hline 10 A 14 ANOS & 38 & 1,2 & 34 & 1,1 & 43 & 1,6 & 46 & 1,7 & 45 & 1,8 & 206 & 1,5 \\
\hline 15 A 19 ANOS & 488 & 15,1 & 504 & 17,0 & 467 & 17,2 & 480 & 17,4 & 438 & 17,3 & 2.377 & 16,7 \\
\hline 20 A 29 ANOS & 1.580 & 48,8 & 1.402 & 47,2 & 1.213 & 44,8 & 1.230 & 44,7 & 1.109 & 43,9 & 6.534 & 46,0 \\
\hline 30 A 39 ANOS & 889 & 27,4 & 832 & 28,0 & 797 & 29,4 & 810 & 29,4 & 735 & 29,1 & 4.063 & 28,6 \\
\hline 40 A 49 ANOS & 245 & 7,6 & 201 & 6,8 & 184 & 6,8 & 185 & 6,7 & 197 & 7,8 & 1.012 & 7,1 \\
\hline 50 A 59 ANOS & 1 & 0,0 & 0 & 0,0 & 4 & 0,1 & 1 & 0,0 & 5 & 0,2 & 11 & 0,1 \\
\hline TOTAL & 3.241 & 100,0 & 2.973 & 100,0 & 2.708 & 100,0 & 2.752 & 100,0 & 2.529 & 100,0 & 14.203 & 100,0 \\
\hline \multicolumn{13}{|c|}{ REGIME DE ATENDIMENTO } \\
\hline Público & 2.684 & 82,8 & 2.639 & 88,8 & 2.481 & 91,6 & 2.705 & 98,3 & 2.515 & 99,4 & 13.024 & 91,7 \\
\hline Privado & 557 & 17,2 & 334 & 11,2 & 227 & 8,4 & 47,0 & 1,7 & 14 & 0,6 & 1.179 & 8,3 \\
\hline TOTAL & 3.241 & 100,0 & 2.973 & 100,0 & 2.708 & 100,0 & 2.752 & 100,0 & 2.529 & 100,0 & 14.203 & 100,0 \\
\hline \multicolumn{13}{|l|}{ ESTABELECIMENTO } \\
\hline MEAC & 1.005 & 38,6 & 713 & 30,0 & 609 & 28,8 & 634 & 28,3 & 415 & 20,7 & 3.376 & 29,8 \\
\hline HDGMM & 606 & 23,2 & 633 & 26,7 & 492 & 23,3 & 418 & 18,6 & 448 & 22,3 & 2.597 & 22,9 \\
\hline HDNSC & 389 & 14,9 & 372 & 15,7 & 377 & 17,9 & 414 & 18,5 & 376 & 18,7 & 1.928 & 17,0 \\
\hline HDGM - BARRA CEARÁ & 284 & 10,9 & 345 & 14,5 & 408 & 19,3 & 464 & 20,7 & 388 & 19,3 & 1.889 & 16,7 \\
\hline HDGM- JOSÉ WALTER & 323 & 12,4 & 311 & 13,1 & 225 & 10,7 & 313 & 14,0 & 380 & 18,9 & 1.552 & 13,7 \\
\hline TOTAL & 2.607 & 100,0 & 2.374 & 100,0 & 2111 & 100,0 & 2243 & 100,0 & 2007 & 100,0 & 11.342 & 100,0 \\
\hline \multicolumn{13}{|c|}{ CARÁTER ATENDIMENTO/ANO } \\
\hline ELETIVO & 306 & 9,4 & 274 & 9,2 & 26 & 0,96 & 48 & 1,74 & 22 & 0,87 & 676 & 4,76 \\
\hline URGÊNCIA & 2.935 & 90,6 & 2.699 & 90,8 & 2.682 & 99,06 & 2.704 & 98,26 & 2.507 & 99,13 & 13.527 & 95,24 \\
\hline TOTAL & 3.241 & 100,0 & 2.973 & 100,0 & 2.708 & 100,0 & 2.752 & 100,0 & 2.529 & 100,0 & 14.203 & 100,0 \\
\hline
\end{tabular}

MEAC-Maternidade Escola Assis Chateaubriand;HDGMM-Hospital Distrital Gonzaga Mota de Messejana;HDNSC- Hospital Distrital Nossa Senhora da Conceição; HDGM/BARRA DO CEARÁ- Hospital Distrital Gonzaga Mota da Barra do Ceará; HDGM/JOSÉ WALTER- Hospital Distrital Gonzaga Mota do José Walter.

Foram registradas 14.203 (100\%) internações por aborto espontâneo, segundo dados de 2010 a 2014; sendo que os anos de maior incidência foram 2010, com 22,8\% (3.241), e 2011, com 20,9\% (2.973). Quanto às faixas etárias de maior ocorrência, foram a de 20 a 29 anos com 46\% (6.534) e a de 30 a 34 anos, com cerca de 28,6\% (4.063). Essas faixas etárias tiveram seus maiores índices no ano de 2010 com $48,8 \%$ (1.580) e $27,4 \%$ (889), respectivamente.

Em relação ao regime de atendimento, ocorreu um maior número de internações no serviço público de saúde, totalizando 92\% (13.024) dos atendimentos, enquanto apenas $8 \%$ (1.179) dos atendimentos foram realizados no serviço privado de saúde. A grande parte de pacientes atendidas por abortos espontâneos aconteceu no ano de 2010, totalizando 3.241 casos (público e privado). O ano de 2014 foi o que teve um maior registro 99,4\% (2.515), seguido pelo ano de 2013 , com 98,3\% (2.705), ambos os anos no serviço público. Já no serviço privado de saúde, durante o mesmo período, foram realizados respectivamente apenas 0,6\% (14) internamentos em 2014, e 1,7\% (47) em 2013

A análise dos dados de internação hospitalar, por aborto espontâneo, no período de 2010 a 2014, nos cinco principais centros de atendimento obstétricos públicos de Fortaleza citados. Um total de 11.342 casos foi levantado. A distribuição dos números de registros do estudo, por instituição pública, evidenciou que: a MEAC ocupa o 1 으 lugar, com 3.376, correspondendo a $29,7 \%$ dos casos dentre as cinco

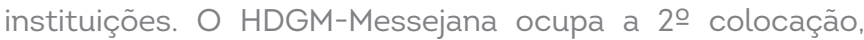
com 2.597 casos, correspondendo a 22,9\%. A seguir, com menores números levantados, segue o HNSC, com 1.928 casos (16,9\%), o HDGM-Barra do Ceará, com 1.889 casos (16,6\%), e por último, o HDGM-José Walter com 1552 (13,6\%). Evidentemente, houve outras instituições públicas que não foram descritas no quantitativo entre os estabelecimentos da pesquisa.

Quanto ao caráter de atendimento, é notório o fato de 
que os atendimentos de urgência estão em maior número, totalizando 95,2\%(13.527), em comparação com apenas 4,76\% (676) eletivos. Os anos com maior número de atendimento de urgência são: 2014, com 2.507 atendimentos, representando $99,13 \%$ do total daquele ano, e 2.704 atendimentos em 2013 , $98,26 \%$ do total do ano.

Gráfico 1: Distribuição do valor médio da internação pósaborto espontâneo. Fortaleza, 2010- 2014.

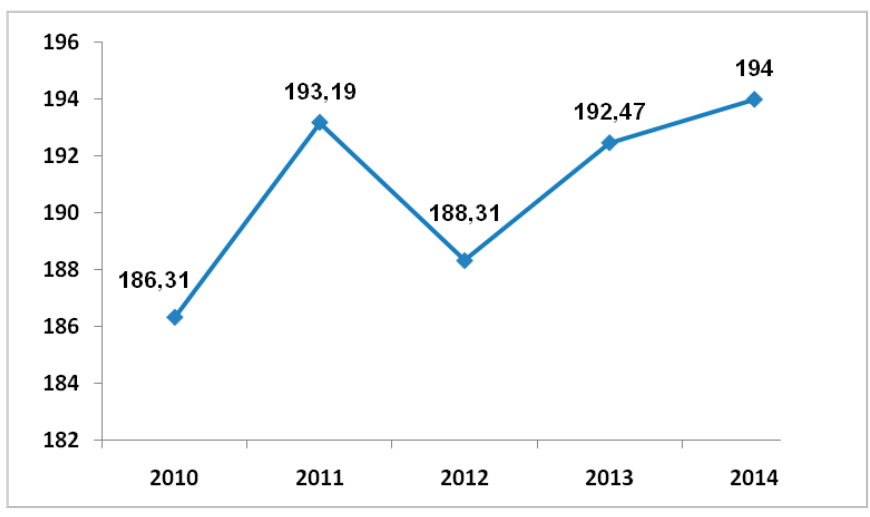

O valor médio da autorização de internamento hospitalar $(\mathrm{AIH})$ variou entre $\mathrm{R} \$ 186,31$ e $\mathrm{R}$ \$194, tendo uma média de $\mathrm{R}$ \$ 190,69 por internação. No período de 2010 a 2011, ocorreu um aumento importante no custo da AIH e, apesar de haver decréscimo de 2011 a 2012, persistiu em elevação o custo de internamento, chegando ao ápice em 2014 com uma média de $\mathrm{R} \$ 194,00$.

O tempo de internamento das mulheres variou de 31 horas e 20 minutos a 36 horas, tendo uma média de 31 horas.

\section{DISCUSSÃO}

A dificuldade em afirmar a real quantidade dos abortamentos espontâneos, dentre todos os abortamentos, decorre muitas vezes pelo fato de algumas mulheres ocultarem a real classificação dos abortos sendo que alguns classificados como espontâneos tenham sido provocados (6).

Quanto à faixa etária, a maior incidência de abortos espontâneos ocorreu entre os 20 a 34 anos, o que pode ser justificado pelo fato de se concentrar o período de maior fecundidade das mulheres, na plenitude de suas atividades sexuais daí estarem mais susceptiveis às ocorrências de gestações desejadas ou não(5).

Houve uma importante redução de $27 \%$ nas internações, por complicações de aborto durante o período de 19952013, fato justificado pela diminuição do número de abortos, incentivo do Ministério da Saúde ao atendimento humanizado, acolhedor e uma atenção de qualidade às mulheres nessa situação(7). Observa-se atualmente uma grande disparidade no número de atendimento no serviço público e privado.
Em um estudo anterior, foi observado que muitas mulheres só procuram os serviços de saúde quando apresentam complicações pós-abortamento. Esse fato pode justificar a maior parte dos atendimentos, realizados entre 2010 e 2014, serem de caráter de urgência ${ }^{(8)}$.

Os números de internação pós-aborto são elevados, causando um problema de saúde pública no Brasi(9). No país, são 250 mil internações por ano para o tratamento de complicações pós-abortamento, internações que geram um custo de 30 milhões de reais ${ }^{(10)}$.

De acordo com a classificação da Organização Panamericana de Saúde e da Organização Mundial da Saúde, a razão da mortalidade materna pode ser estratificada em: baixa (menor de 20 óbitos por 100.000 nascidos vivos), média (entre 20 a 49 óbitos por 100.000 nascidos vivos), alta (entre 50 a 149 óbitos por 100.000 nascidos vivos) e muito alta (maior ou igual a 150 óbitos por 100.000 nascidos vivos).

A partir de dados levantados do boletim epidemiológico da Secretaria de Saúde do Estado do Ceará sobre mortalidade materna é possivel afirmar que o número de óbitos, por abortamento, vem sendo reduzido em nosso estado. Entre 2005 e 2009, foram notificados 27 óbitos por 100.000 nascidos vivos. O aborto foi apontado como a terceira principal causa obstétrica direta de óbito materno nesse período. Já de 2010 a 2013, foram notificados 15 óbitos por 100.000 nascidos vivos, representando apenas $5,7 \%$ dos casos registrados. Neste estudo, de acordo com os dados do DATASUS, foram registrados apenas dois óbitos, sendo um em 2012 e um em $2013^{(10)}$

O tempo de internamento das mulheres evidencia o que já foi relatado em estudo anterior, no qual $86 \%$ permaneceram um dia, enquanto que $14 \%$ das internações têm média superior a um dia ${ }^{(11)}$. Os custos, para o Sistema Único de Saúde, com internações por abortamento espontâneo são muito reduzidos, já que a maior parte deles não exige internação, e as complicações são predominantemente resultantes de abortos provocados ${ }^{(12)}$.

Quanto aos custos com a AIH, existe a necessidade de um estudo mais detalhado do motivo que levou ao decréscimo dos valores de 2011 a 2012 e, em seguida, uma nova elevação em 2014. Faz-se necessário entender se essa elevação de 2012 a 2014 corresponde apenas a um aumento da população em Fortaleza, especialmente a feminina em período fértil, à desatenção com programas educacionais relativos ao assunto estudado ou apenas a um reajuste realizado no valor das internações.

\section{CONCLUSÃO}

O estudo retrata o perfil epidemiológico das ocorrências de internações por aborto espontâneo no município de Fortaleza no período de 2010 a 2014. Foi possivel observar na 
pesquisa que a grande maioria dos atendimentos concentrase no sistema público de saúde, com 91,7\% dos registros. Entre os estabelecimentos, a MEAC atendeu 29,8\% dos casos; desses, 95,2\% foi em caráter de urgência, e gastou um valor médio de $\mathrm{R} \$ 190,69$ por internação.

É importante mencionar que os dados nos permitem afirmar a real urgência do acesso da população à educação sexual nas escolas, a partir da faixa etária em que os adolescentes iniciam suas atividades sexuais, para que seja possivel o amadurecimento da importância da gravidez com todas as suas circunstâncias e complicações. Outro ponto a ser abordado deve ser a ênfase na importância do pré-natal, uma vez diagnosticada a gravidez, podendo ser introduzida uma cartilha educativa.
O fato de possivelmente haver ocultação da real causa do abortamento (espontâneo ou provocado) empobrece a realidade dos dados, bem como as fontes que são utilizadas para a obtenção dos mesmos, consistindo em um viés de suma importância.

Esses dados também mostram a necessidade da implantação de protocolos de orientação escolar, como preventivo de gravidez indesejada ou precoce, e nas unidades médico-hospitalares específicas como esclarecimento causal às pacientes que são recebidas com quadro de abortamento espontâneo. A condensação de todos esses dados poderá ser notificada a uma central pública para geração de estudos mais pertinentes e próximos da realidade de cada território (municipal, estadual e nacional)

\section{REFERÊNCIAS}

1. Wiese IRB, Saldanha AAW. Aborto induzido na interface da saúde e do direito. Saúde Soc. 2014;23(2):536-547.

2. Borsari CMG, Nomura RMY, Benute GRG, Lucia MCS, Francisco RPV Zugaib M. Aborto provocado em mulheres da periferia da cidade de São Paulo: vivência e aspectos socioeconômicos. Rev Bras Ginecol Obstet. 2013 jan;35(1):27-32. DOI http://dx.doi.org/10.1590/s010072032013000100006

3. Cúnico SD, Faraj SP, Quintana AM, Beck CLC. Algumas considerações acerca da legalização do aborto no Brasil. Mudanças, Psicol da Saúde. 2014 jan-jun;22(1):41-47. DOI http://dx.doi.org/10.15603/2176-1019/mud. v22nlp4l-47.

4. Ribeiro Filho JF, Ribeiro LS, Machado PHF, Machado TMG. Perfil das mulheres submetidas à curetagem uterina pós-abortamento em um hospital público. Rev Gest Saúde. 2015;6(2):1354-1366.

5. Ribeiro TB, Bolpato MB. Aborto provocado: consequências físicas, psicológicas e as implicações para a assistência de enfermagem. Revista Eletrônica da Univar. 2013:1(9):136-141.

6. Silva MM, Castro PM, Theisen C, Freitas HMB, Colomé JS. Problemática do aborto em Santa Maria, Rio Grande do Sul: dados epidemiológicos. Discip Sci, Cienc Saude. 2014;15(2):249-259.

7. Castro F. Número de abortos no Brasil teve redução de $26 \%$ nos últimos 20 anos, segundo estudo [Internet]. Estadão. 2015 ago 21 [acesso em 2016 set 2]. Disponivel em: http://ciencia.estadao.com.br/noticias/geral,brasilteve-queda-de-26-no-numero-de-abortos-nos-ultimos-20-anos--dizestudo,1748631.

8. Diniz D, Medeiros M. Itinerários e métodos do aborto ilegal em cinco capitais brasileiras. Ciên Saúde Colet. 2012:17(7):1671-1681.

9. Alves LF. Aborto provocado, aspectos éticos e legais: o direito a vida.
Webartigos [Internet]. 2009 [acesso em 2016 out 6]. Disponivel em: http:// www.webartigos.com/artigos/aborto-provocado-aspectos-eticos-elegais-o-direito-a-vida/16892.

10. Garcia M. Consenso para quê? Revista Ciência Hoje [Internet]. 2013 [acesso em 2016 out 6]. Disponivel em: http://www.cienciahoje.org.br/ noticia/v/ler/id/2494/n/consenso_para_que.

11. Ceará, Governo do Estado do Ceará, Secretaria da Saúde. Informe epidemiológico mortalidade materna. [Internet]. Fortaleza; 2015 [acesso em 2015 maio 16]. Disponivel em: file:///C:/Users/Camila/Downloads/ informe mortalidade materna marco 2014\%20.pdf.

12. Benute GRG, Nonnenmacher D, Nomura RMY, Lúcia MCS, Zugaib M. Influência da percepção dos profissionais quanto ao aborto provocado na atenção à saúde da mulher. Rev Bras Ginecol Obstet. 2012;34(12):69-73.

13. Borges APG. Abortamento espontâneo: um estudo epidemiológico [dissertação] [Internet]. Bragança: Instituto Politécnico de Bragança; 2013. [acesso em 2015 nov 19]. Disponivel em: http://bibliotecadigital.ipb.pt/ bitstream/10198/9201/1/Amandina\%20Borges.pdf.

14. SUS atende 100 vezes mais casos pós-aborto do que faz interrupções legais [Internet]. São Paulo; 2016. [acesso em 2016 out 6]. Disponivel em: http://noticias.uol.com.br/cotidiano/ultimas-noticias/2016/03/10/susatende-100-vezes-mais-casos-pos-aborto-do-que-faz-interrupcoeslegais.htm.

15. Brasil, Ministério da Saúde. DATASUS: Departamento de Informática do SUS. [Internet]. Brasilia; 2007. [acesso em 2016 out 6]. Disponivel em: http:// datasus.saude.gov.br/informacoes-de-saude/tabnet.

16. Brito RS, Santos DLA, Silva AB. Perfil de mulheres em situação de abortamento internadas em uma maternidade escola. Rev enferm UFPE on line. [Internet]. 2015 [acesso em 2016 out 6];9(1):15-22. Disponivel em: file:///C:/Users/ Camila /Downloads/6747-66643-1-PB\%20(6).pdf 